\section{Pregnancy in a liver transplant patient treated with everolimus and tacrolimus}

\section{To the Editor,}

We appreciated the paper regarding the quality of life in patients after liver transplantation (LT) by Domingos et al. [1], recently published in the Journal of Gastrointestinal and Liver Diseases. The authors reported a similar or superior quality of life in LT patients compared to the general population. Due to advances in immunology and transplant medicine, women with a history of LT could have successful pregnancies. However, data on pregnancy outcomes is still limited, especially for women with a history of liver graft failure, receiving the mammalian target of rapamycin (mTOR) inhibitors. Recently, many studies focused on everolimus benefits in the immunosuppression regimen after LT, which might be initiated early post-LT or in later monitoring phases, allowing the calcineurin inhibitor reduction [2]. Published reports on pregnant women exposed to tacrolimus documented no increased risk of malformations, but data on pregnancy under everolimus is still relatively scarce $[3,4]$.

We report a good clinical outcome of an unplanned pregnancy in a LT female patient aged 30 under everolimus and tacrolimus immunosuppression therapy.

The patient had a successful LT at 20-year old for decompensated liver cirrhosis caused by Wilson disease. The recipient received tacrolimus as immunosuppressive therapy for 5 years with stable graft liver function. In 2016, at 25-years old, she had an unplanned pregnancy discovered at 8 weeks of gestation. The immunosuppressive therapy was reduced and then completely stopped by the patient herself, during the second semester of pregnancy. At 27 weeks of pregnancy she was admitted in the hospital with high levels of bilirubin (total bilirubin $10.7 \mathrm{mg} / \mathrm{dl}$, conjugated bilirubin $9 \mathrm{mg} / \mathrm{dl}$, upper normal value $1 \mathrm{mg} / \mathrm{dl}$ ), mild cholestasis and elevated aminotransferase levels ( 8 times the upper normal limit). The liver biopsy depicted mild mononuclear inflammatory infiltrate in the portal space with disappearance of bile ducts and perivenular mononuclear inflammation in the terminal hepatic venules, corresponding to chronic liver graft rejection. The patient received tacrolimus and pulse therapy with solumedrol, with no benefit. She had a cesarean section at 29 weeks of pregnancy (the newborn presented Apgar score 7, respiratory distress syndrome, and no congenital malformations). The chronic rejection was successfully managed after delivery, by adding $2 \mathrm{mg}$ of everolimus (blood trough levels 3-8 $\mathrm{ng} / \mathrm{mL}$ ) to tacrolimus (blood trough levels of 3-5ng/ml).

In 2020 she had the second unplanned pregnancy, discovered at 7 weeks. The patient was carefully monitored by the obstetrician and the hepatologist. She received lower doses of everolimus $(1.5 \mathrm{mg} /$ day and decreased to $1 \mathrm{mg} /$ daily from the 28 weeks of gestation) and lower doses of tacrolimus (4.5 mg/day), with normal liver graft function. Ultrasound monitoring did not detect any fetus abnormalities. Considering her history of chronic graft liver rejection, a cesarean section was scheduled at 34 weeks to balance between the mother risk for graft rejection and the optimal time for fetus delivery. The newborn weighted 2,250 g had an Apgar score 8 and presented respiratory distress syndrome with respiratory failure for which surfactant administration and non-invasive respiratory support were ensured; no clinical signs of malformations were reported. The patient and her newborn were in good clinical condition at 3 months follow-up.

Although mTOR inhibitors have been reported to increase the fetus mortality in experimental animals, no teratogenic effects have been seen either in rats or rabbits [3]. As the teratogenic risk cannot be ruled out, the guidelines recommend that another drug be used as a substitute for mTOR inhibitors. The antiproliferative effect of mTOR inhibitors might prevent maturing of the fetus [4]. The mTOR signaling in the fetal liver, trophoblast, and decidua serves as a critical hub in the overall homeostatic control of fetal growth [5]. In the fetal liver mTORs regulate the secretion and phosphorylation of insulinlike growth factor binding protein 1 , thereby controlling the bioavailability of growth hormones [5]. In the placenta, mTORs respond to a large number of growth-related signals and in the maternal compartment, mTORs are an integral part of 
a decidual nutrient sensor, connecting oxygen and nutrient availability to the growth hormones. Based on these scientific reports our patient was exposed to lower doses of everolimus during the second pregnancy.

This presentation reports a good clinical outcome for a newborn exposed to everolimus and tacrolimus immunosuppressive therapy for LT.

\section{Razvan Ciortea ${ }^{1,2}$, Lidia Ciobanu ${ }^{1,3}$, Emil Matei ${ }^{1,4}$, Chiara Zanfi ${ }^{5}$, Ligia Daniela Blaga ${ }^{1,2}$ \\ 1) Iuliu Hatieganu University of Medicine and Pharmacy, Cluj- Napoca, Romania; 2) $2^{\text {nd }}$ Obstetrics and Gynecology Department, County Hospital, Cluj-Napoca, Romania; 3) Regional Institute of Gastroenterology and Hepatology, Cluj-Napoca, Romania; 4) Fundeni Clinical Institute, Bucharest, Romania; 5) Multiorgan Transplantation Department, S. Orsola-Malpighi Hospital, University of Bologna, Bologna, Italy}

Correspondence: Lidia Ciobanu, lidia.ciobanu@umfcluj.ro

Conflicts of interest: None.

DOI: $10.15403 /$ jgld-3181

\section{REFERENCES}

1. Domingos MF, Coelho JCU, Nogueira IR, et al. Quality of Life after 10 Years of Liver Transplantation. J Gastrointestin Liver Dis 2020;29:611 616. doi:10.15403/jgld-2829.

2. De Simone P, Nevens F, De Carlis L, et al; H2304 Study Group. Everolimus with reduced tacrolimus improves renal function in de novo liver transplant recipients: a randomized controlled trial. Am J Transplant 2012;12:3008-3020. doi:10.1111/j.1600-6143.2012.04212.x

3. Leroy C, Rigot JM, Leroy M, et al. Immunosuppressive drugs and fertility. Orphanet J Rare Dis 2015;10:136. doi:10.1186/s13023-0150332-8

4. Ponticelli C, Moroni G. Fetal Toxicity of Immunosuppressive Drugs in Pregnancy. J Clin Med 2018;7:552. doi:10.3390/jcm7120552

5. Gupta MB, Jansson T. Novel roles of mechanistic target of rapamycin signaling in regulating fetal growth. Biol Reprod 2019;100:872-884 doi:10.1093/biolre/ioy249

\section{The use of a proton pump inhibitor does not increase the risk of colorectal cancer: a meta- analysis}

\section{To the Editor,}

The association between proton pump inhibitors (PPIs) use and the risk of colorectal cancer (CRC) is controversial in literature [1-10]. We summarize the existing evidence on the risk of CRC in patients with PPIs use by conducting a meta-analysis.

We systematically searched PubMed, Embase and Web of science for studies evaluating the association of PPIs use with the risk of CRC until July 2020. The following key words were used: 'proton pump inhibitor', 'PPI', 'lansoprazole', 'dexlansoprazole', 'esomeprazole, 'pantoprazole', 'omeprazole', rabeprazole, 'colon cancer,' 'colon neoplasm, 'colon tumor', 'colon carcinoma', 'rectal cancer', 'rectal neoplasm', 'rectal tumor', 'rectal carcinoma', 'colorectal cancer', 'colorectal neoplasm', 'colorectal tumor', 'colorectal carcinoma'. Boolean operators, such as 'OR' and 'AND', were used to combine the above keywords. No language restrictions and manually search for references in included studies.

The following content criteria were used to select studies for inclusion in the meta-analysis: (1) the association of PPIs use with the risk of CRC was evaluated; (2) sufficient data to allow calculation of odds ratio (OR) with $95 \%$ confidence interval (CI) was provided. Studies with insufficient data were excluded. Sensitivity analysis was conducted by excluding each study in turn. Egger's test quantified the publication bias.

A total of 3,431 articles were initially collected. After removing 1,059 duplicates, 2,372 articles remained. After screening title and abstract, ten articles that explored the relationship between PPI use and CRC risk published between 2007 and 2020 were selected [1-10]. Among them, eight were published in full text $[1,3,4,6-10]$, one was a conference abstract [5], and another was a letter [2]. All included articles underwent a qualitative and quantitative assessment. The basic information of included articles is displayed in Supplementary Table I.

We identified three cohort and seven case-control studies including 1,069,716 participants (Supplementary Table I). Eight studies were conducted in Europe and America, and two in Asia. Pooled results showed that there was no obvious association of PPIs use with the risk of CRC (OR 1.22, 95\%CI 0.93-1.62, $\mathrm{p}=0.154$ ) (Fig. 1). The results did not differ significantly from the overall analysis based on sensitivity analysis, revealing that the outcome was stable. No significant bias was detected ( $p=0.918$, Egger's test). The subgroup analysis [study type (cohort study or case-control study), sample size ( $>50000$ or $\leq 50000$ ), cumulative use of PPIs ( $>5$ year or $\geq 10$ years)] showed the ORs consistent with the overall analysis. The 95\%CI lower limit of combined OR gradually approached 1 with the extension of medication time (duration of PPIs use $\geq 10$ years: OR 1.11, 95\%CI 0.84-1.46; duration of PPIs use $>$ 5years: OR $1.25,95 \% \mathrm{CI} 0.80-1.97)$, which may indicate a gradual increased risk of CRC.

There are several limitations in our meta-analysis. Firstly, all included studies are retrospective. Secondly, considering the heterogeneity among the meta-analysis, the results should be treated with caution. Thirdly, most of the patients are from Europe and America. Finally, no adjustment was performed concerning the family history of CRC, which is one of the most important risk of CRC.

Despite these limitations, the meta-analysis also has some advantages. Firstly, our study included more than four times as many patients as previous meta-analyses $[11,12]$, and the results had higher statistical power and were more persuasive. Secondly, based on sensitivity analysis the results were stable. Furthermore, there was no possible publication bias. More importantly, we found that the risk of CRC might increase with prolonged PPIs use.

In conclusion, this meta-analysis reveals that use of PPIs does not increase the risk of CRC, which is consistent with the other two studies $[11,12]$. However, the risk of CRC may gradually increase with long-term PPI use. CRC is a 


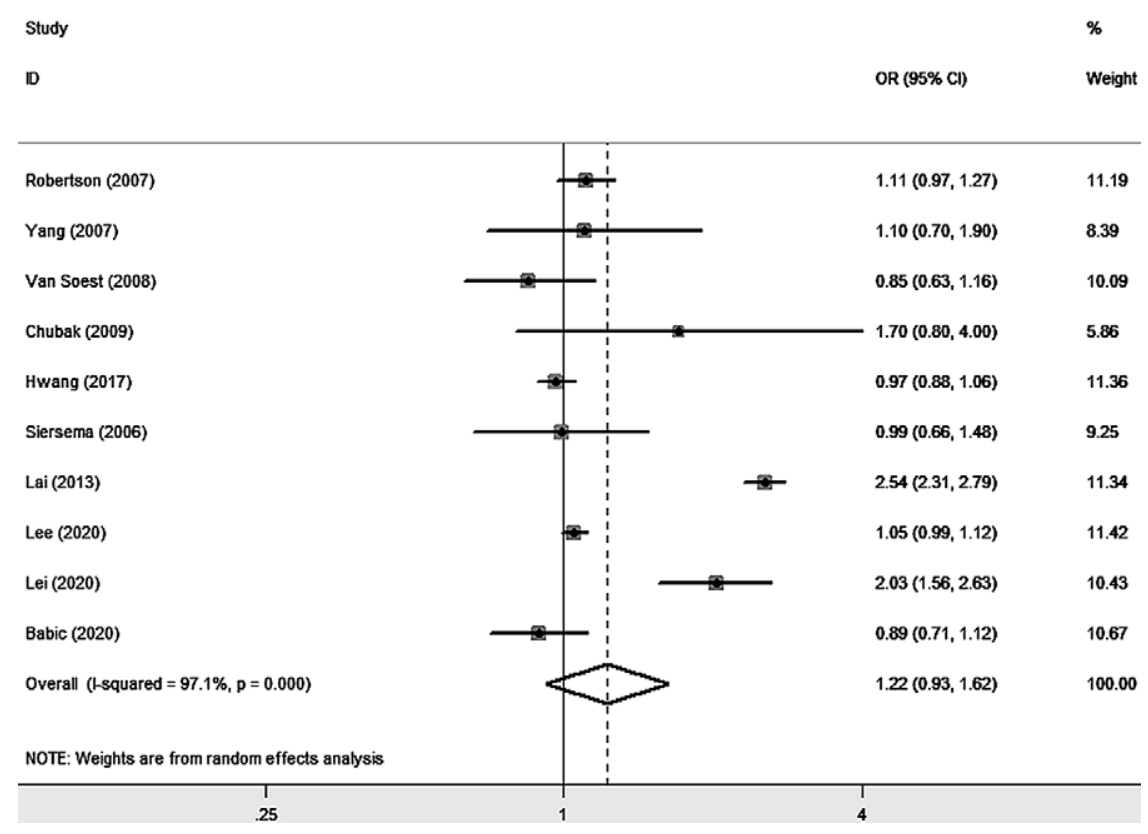

Fig. 1. Forest plot of the association between PPI use and CRC risk.

progressive disease that takes many years from the initiation to the progression to cancer. Therefore, with the extension of PPIs use time, people would expect to see an increased CRC risk become obvious. Multi-area randomized controlled studies are required to confirm our results.

\section{Rongqiang $\mathrm{Liu}^{1}$, Yi Shao ${ }^{2}$}

1) Department of hepatobiliary surgery, The First Affiliated Hospital of Guangzhou Medical University, Guangdong; 2) Department of Ophthalmology, The First Affiliated Hospital of Nanchang University, Jiangxi, China

Correspondence: Yi Shao, freebee99@163.com

\section{Conflicts of interest: None.}

Acknowledgements: This study was supported by the National Natural Science Foundation of China (No:81660158); Natural Science Key Project of Jiangxi Province (No: 20161ACB21017); Youth Science Foundation of Jiangxi Province (No: 20151BAB215016, 20161BAB215198). The funders had no role in the study design, data collection and analysis, decision to publish, or preparation of the manuscript.

DOI: $10.15403 /$ jgld-2878

Supplementary material: To access the supplementary material visit the online version of the J Gastrointestin Liver Dis at http://dx.doi. org/10.15403/jgld-2878

\section{REFERENCES}

1. Chubak J, Boudreau DM, Rulyak SJ, Mandelson MT. Colorectal cancer risk in relation to use of acid suppressive medications. Pharmacoepidemiol Drug Saf 209;18:540-544. doi:10.1002/pds.1749

2. Lai SW, Liao KF, Lai HC, Lin CL, Sung FC. Use of proton pump inhibitors correlates with increased risk of colorectal cancer in Taiwan. Asia Pac J Clin Oncol 2013;9:192-193. doi:10.1111/ajco.12054
3. Hwang IC, Chang J, Park SM. Emerging hazard effects of proton pump inhibitor on the risk of colorectal cancer in low-risk populations: A Korean nationwide prospective cohort study. PLoS One 2017;12:e0189114. doi:10.1371/journal.pone.0189114

4. Robertson DJ, Larsson H, Friis S, Pedersen L, Baron JA, Sørensen HT. Proton pump inhibitor use and risk of colorectal cancer: a populationbased, case-control study. Gastroenterology 2007;133:755-760. doi:10.1053/j.gastro.2007.06.014

5. Siersema PD, Yu S, Sahbaie P, et al. Colorectal neoplasia in veterans is associated with Barrett's esophagus but not with proton-pump inhibitor or aspirin/NSAID use. Gastrointest Endosc 2006;63:581-586. doi:10.1016/j.gie.2005.08.043

6. van Soest EM, van Rossum LG, Dieleman JP, et al. Proton pump inhibitors and the risk of colorectal cancer. Am J Gastroenterol 2008;103:966-973.

7. Yang YX, Hennessy S, Propert K, Hwang WT, Sedarat A, Lewis JD. Chronic proton pump inhibitor therapy and the risk of colorectal cancer. Gastroenterology 2007;133:748-754. doi:10.1053/j.gastro.2007.06.022

8. Lee JK, Merchant SA, Schneider JL, et al. Proton Pump Inhibitor Use and Risk of Gastric, Colorectal, Liver, and Pancreatic Cancers in a Community-Based Population. Am J Gastroenterol 2020;115:706-715. doi:10.14309/ajg.0000000000000591

9. Lei WY, Wang JH, Yi CH, et al. Association between use of proton pump inhibitors and colorectal cancer: A nationwide population-based study. Clin Res Hepatol Gastroenterol 2020 Mar 26. doi:10.1016/j. clinre.2020.02.017. Epub ahead of print.

10. Babic A, Zhang X, Morales-Oyarvide V, et al. Acid-suppressive medications and risk of colorectal cancer: results from three large prospective cohort studies. Br J Cancer 2020;123:844-851. doi:10.1038/ s41416-020-0939-y

11. Chen S, Song X, Gao X, et al. Proton pump inhibitors and the risk of colorectal cancer: a meta-analysis. J Clin Gastroenterol 2011;45:177. doi:10.1097/MCG.0b013e3181ea181e

12. Ahn JS, Park SM, Eom CS, Kim S, Myung SK. Use of Proton Pump Inhibitor and Risk of Colorectal Cancer: A Meta-analysis of Observational Studies. Korean J Fam Med 2012;33:272-279. doi:10.4082/kjfm.2012.33.5.272 


\section{Micro-elimination of hepatitis $\mathrm{C}$ virus infection - the beginning of the end}

\section{To the Editor,}

Hepatitis $\mathrm{C}$ virus (HCV) is one of the leading causes of chronic liver disease and liver-related deaths, worldwide [1]. The revolution in HCV treatment with direct acting antiviral agents (DAAs) since 2014 has entirely changed the landscape of $\mathrm{HCV}$ therapy. A disease that was cured only in less than half of patients with a long therapy and with side effects is now a disease that can be eradicated in 8-12 weeks, almost in all patients, with all-oral interferon free regimens based on DAAs. Despite this triumphal therapeutic event, HCV still is a global concern, affecting around 71 million people worldwide [1]. This epochal therapeutic progress of HCV through DAAs has led to an immense international interest for global elimination of $\mathrm{HCV}$ as a public health threat and to the ambitious decision of the World Health Organization (WHO) in 2016 to eliminate viral hepatitis by 2030 (90\% reduction in incidence, treatment of $80 \%$ patients who are eligible and $65 \%$ reduction in mortality) [1].

Romania is considered the country with the highest prevalence of $\mathrm{HCV}$ in Europe, with various reported figures in the last decades $(5.9 \%$ in $1990,3.23 \%$ in 2010 , recently estimated 1.39\%) [2-4]. Moldavia is a Romanian province with the highest prevalence of $\mathrm{HCV}$ [3].

To fulfil the WHO targets each country should have its individual plan as there is no "one size-fits-all" approach [5]. Thus, several strategies have been proposed. One is microelimination, a concept that breaks down the national elimination goals into smaller, easier to accomplish tasks such as specific subpopulations (prisoners, people with haemophilia or HIV, hemodialysis, transplant recipients, people who inject drugs, men who have sex with men) as well as geographical areas (regions, cities, villages). The community-based strategy combined educational campaign, testing and treatment [4]. We adopted such a strategy in a project designed to educate, test and treat HCV with the aim of eliminating HCV infection in all adults, in a village named Vetrisoaia, located in Eastern Romania, in the Moldavia province, Vaslui county, a region considered as having a poor socioeconomic level, with difficult access to healthcare system. A mobile team of gastroenterologists, residents and nurses from the Institute of Gastroenterology and Hepatology, St. Spiridon Hospital Iasi was created and undertook community mobilization with the aid of the local leadership (mayor, village council, general practitioners, teachers, priest). Our project was carried out between $1^{\text {st }}$ March 2019-31 ${ }^{\text {st }}$ December 2019. The adult population of Vetrisoaia, comprising 3,507 people were invited to be tested by direct communication, house-to-house. For certain situations, the screening was organized at a household level, this being one of the reasons for the high coverage of the village.

The screening was made using rapid diagnostic orientation tests: $2,945(84 \%)$ subjects were tested and 78 (2.64\%) were found with positive HCV antibodies and were scheduled for further evaluation in our hospital, in order to be linked to care. Out of sixty-six (85\%) presented for evaluation, 55 (83\%) had HCV RNA detectable and 25\% had advanced fibrosis (F3/F4). Fifty-four (98\%) completed antiviral treatment and 53 (99\%) obtained sustained virological response (SVR).
To our knowledge this project is the first in Romania and among only a few in Europe. A similar project was performed in Egypt [6]. This proof-of-concept study could inspire similar projects in Romania and other countries, which have a high percentage of rural populations.

Laura Huiban ${ }^{1,2}$, Carol Stanciu ${ }^{1}$, Catalin Sfarti ${ }^{1,2}$, Cristina Muzica ${ }^{1,2}$, Tudor Cuciureanu ${ }^{1,2}$, Sebastian Zenovia ${ }^{1,2}$, Georgiana Frunzuc ${ }^{2}$, Ioana Damian ${ }^{2}$, Anca Trifan ${ }^{1,2}$

1) Grigore T. Popa University of Medicine and Pharmacy, Department of Gastroenterology, Iasi; 2) Institute of Gastroenterology and Hepatology, Iasi, Romania

Correspondence: Carol Stanciu, stanciucarol@yahoo.com

Conflicts of interest: None.

Acknowledgements: This work was supported by an unrestricted competitive Abbvie Company and the patient association "Equity in health". They had no role in the collection, analysis or interpretation of the data, as well as in the preparation, review or approval of the manuscript. DOI: $10.15403 /$ jgld-3028

\section{REFERENCES}

1. Lombardi A, Mondelli MU; ESCMID Study Group for Viral Hepatitis (ESGVH). Hepatitis C: Is eradication possible? Liver Int 2019;39:416426. doi:10.1111/liv.14011

2. Molnar GB, Popa S, Jebeleanu L, Damian C. Studiul prevalentei markerilor serici ai infectiei cu virusurile hepatitelor in anamneza epidemiologica a populatiei. Bacteriol Virusol Parazitol Epidemiol 1994;30:141-150.

3. Gheorghe L, Csiki IE, Iacob S, Gheorghe C, Smira G, Regep L. The prevalence and risk factors of hepatitis $\mathrm{C}$ virus infection in adult population in Romania: a nationwide survey 2006 - 2008. J Gastrointestin Liver Dis 2010;19:373-379.

4. Gheorghe L, Iacob S, CsikiIE, et. al. The Prevalence of HCV Infection and Risk Factors in a Hospital Based Population Screening, a First Step to the Micro-Elimination of HCV Infection in Medical Institutions from Romania - Results of the HepC ALERT Study. J Gastrointestin Liver Dis 2020;29:587-593. doi:10.15403/jgld-3084

5. Lopes H, Baptista-Leite R, Franco D, et al. Modeling the Puzzle of Hepatitis C Epidemiology in Romania: A Pathway to Control. J Gastrointestin Liver Dis 2020;9;29:377-384. doi:10.15403/jgld-643

6. Shiha G, Metwally AM, Soliman R, Elbasiony M, Mikhail NNH, Easterbrook P. An educate, test, and treat programme towards elimination of hepatitis $\mathrm{C}$ infection in Egypt: a community-based demonstration project. Lancet Gastroenterol Hepatol 2018;3:778-789. doi:10.1016/S2468-1253(18)30139-0

\section{Liver injury in COVID-19 patients - a multidisciplinary experience and a call for national consensus}

\section{To the Editor,}

We read with considerable interest the recent detailed systematic review and meta-analysis by Xin et al. [1] published 
in the Journal of Gastrointestinal and Liver Diseases, on the abnormal liver function tests of patients with coronavirus disease 2019 (COVID-19) in Mainland China. In their study, the severity of COVID-19 was associated with liver damage and could be a risk factor for abnormal liver function tests (LFTs). We also focus on the impact of abnormal liver function on the prognosis of COVID-19, by presenting a multidisciplinary approach.

By the end of September 2020, COVID-19 had affected more than 31 million patients worldwide, with a mortality rate of approximately $4 \%$ [2] and still represents an important medical challenge for physicians worldwide $[3,4]$.

We performed a retrospective, single-centre, observational study of 1,479 patients confirmed with COVID-19, admitted to Mureș County Clinical Hospital, designated as a support hospital, in three different Departments: Gastroenterology, Infectious Diseases and Intensive Care Unit. The observed patients were admitted during a 6-month period (March 2020-August 2020). We analysed clinical features, laboratory tests, medications, comorbidities, clinical outcomes such as intensive care unit admission, discharge, death, and length of hospital stay. Adult patients, with a laboratory confirmation of COVID-19, irrespective of clinical signs and symptoms, were included in the study. Pre-existing liver disease with a documented history of abnormal LFTs was the main exclusion criteria.
From a total of 1,479 patients admitted in Mureș County Clinical Hospital, 146 patients (9.87\%) which presented with abnormal LFTs at admission or during the first 7 days of hospitalisation were included in the study. Mean age was 48.68 years (46.54-50.82), with a male: female ratio of 1.43:1. No deaths were reported in our inclusion group compared to the total number of deaths of 85 (5.74\%).

Paraclinical characteristics of patients in the severe COVID-19 and non-severe groups, as well as those of patients admitted to the intensive care unit are shown in Table I. During hospitalisation, the majority of patients $(68.4 \%)$ presented with mild liver injury and median duration of liver injury was 5 days. None of the patients with severe liver injury expressed normal liver function at discharge, and the predominant mechanism of severe acute respiratory syndrome coronavirus 2 (SARS-CoV-2) related liver injury was hepatocellular (59.5\%). The average length of hospital stay was 16.21 days (standard deviation 7.43) and was not statistically longer in patients with severe liver injury.

Consistent with Xian et al. [1] results, this study has found that severe COVID-19 patients, presented early onset of liver injury, and a slow decrease of abnormal LFTs, with a lack of normalization of LFTs at discharge. Moderate to severe liver injury was more common in patients with severe COVID19, admitted to the intensive care unit. More studies are required

Table I. Demographics and paraclinical characteristics of 146 enrolled COVID-19 patients.

\begin{tabular}{|c|c|c|c|c|c|c|}
\hline \multirow[t]{2}{*}{ Parameter } & \multicolumn{3}{|c|}{ Severity of COVID-19 } & \multicolumn{3}{|c|}{ Intensive care unit admission } \\
\hline & Non-severe $n=130$ & Severe $n=16$ & $\mathrm{p}$-value & No $n=136$ & Yes $n=10$ & $\mathrm{p}$-value \\
\hline Age (years) & $47.73 \pm 13.41$ & $56.5 \pm 9.08$ & 0.012 & $48.35 \pm 13.38$ & $53.4 \pm 11.07$ & 0.246 \\
\hline Length of hospitalization (days) & $15.93 \pm 7.67$ & $18.63 \pm 5.76$ & 0.177 & $15.8 \pm 7.48$ & $22 \pm 5.42$ & 0.007 \\
\hline Hemoglobin (g/dL) & $13.69 \pm 1.46$ & $13.79 \pm 2.69$ & 0.802 & $13.68 \pm 1.52$ & $13.985 \pm 2.76$ & 0.571 \\
\hline ALT at admission (U/L) & $97.15 \pm 83.13$ & $83.13 \pm 68.89$ & 0.518 & $94.75 \pm 81.09$ & $107.4 \pm 91.96$ & 0.638 \\
\hline ALT highest value (U/L) & $160.22 \pm 167.02$ & $162.06 \pm 69.36$ & 0.965 & $148.52 \pm 97.07$ & $322.3 \pm 485.03$ & 0.001 \\
\hline AST at admission (U/L) & $62.39 \pm 56.57$ & $62.25 \pm 39.49$ & 0.992 & $60.88 \pm 54.96$ & $82.8 \pm 51.61$ & 0.224 \\
\hline $\mathrm{TB}(\mathrm{mg} / \mathrm{dL})$ & $0.91 \pm 0.34$ & $0.98 \pm 0.39$ & 0.462 & $0.9 \pm 0.32$ & $1.11 \pm 0.49$ & 0.092 \\
\hline APTT (sec) & $24.36 \pm 7.75$ & $22.4 \pm 3.32$ & 0.42 & $24.12 \pm 7.6$ & $22.96 \pm 3.46$ & 0.659 \\
\hline INR & $1.06 \pm 0.15$ & $1.07 \pm 0.13$ & 0.831 & $1.06 \pm 0.14$ & $1.11 \pm 0.13$ & 0.326 \\
\hline Urea (mg/dL) & $36.27 \pm 14.85$ & $55.33 \pm 24.92$ & $<0.001$ & $37.26 \pm 16.52$ & $54.41 \pm 20.99$ & 0.003 \\
\hline Creatinine (mg/dL) & $0.84 \pm 0.21$ & $0.92 \pm 0.24$ & 0.196 & $0.85 \pm 0.21$ & $0.94 \pm 0.24$ & 0.213 \\
\hline Sodium (mmol/L) & $138.72 \pm 3.43$ & $137.5 \pm 4.68$ & 0.204 & $138.69 \pm 3.49$ & $137.2 \pm 4.85$ & 0.211 \\
\hline Potassium(mmol/L) & $4.35 \pm 0.51$ & $4.23 \pm 0.65$ & 0.399 & $4.31 \pm 0.51$ & $4.69 \pm 0.61$ & 0.027 \\
\hline Glycemia (mg/dL) & $120.41 \pm 54.79$ & $142.69 \pm 73.58$ & 0.143 & $118.04 \pm 51.62$ & $187.9 \pm 87.97$ & 0.003 \\
\hline
\end{tabular}

Abbreviations: ALP; alkaline phosphatase; ALT: alanine aminotransferase; AST: aspartate aminotransferase; COVID-19: coronavirus disease 2019; GGT: gamma glutamyl transferase; INR: international normalized ratio; TB: total bilirubin. 
in order to corelate and establish the pathogenesis of LFTs abnormality in patients with a mild form of the disease.

In June 2020, EASL-ESCIMD provided a consensus position paper [5] regarding the approach and management of patients with pre-existing liver disease, taking into consideration the fact that they represent a vulnerable cohort of patients. Our study emphasises the need for a consensus regarding the type of approach and management of SARSCOV2 liver injury, as well as the need to follow up patients without any known or manifest pre-existing liver disease, but who present with abnormal LFTs associated with COVID- 19. Thus, the challenge of hepatologists in the future will be to predict and prevent any other additional source of liver injury in COVID-19 patients.

Standardized definitions and guidelines of liver damage management in COVID-19 are required for consultant hepatologists in order to clarify differential diagnosis and to apply a unitary measure of care [6].

Etiological investigations should be performed at an early stage in COVID-19 liver damage, especially in patients with a length of disease higher than 7 days, or in patients who present a rapid decrease of SARS-COV2 viral load. Close followup of patients with SARS-COV2 liver injury should not be disregarded, even after the normalization of LFTs. A national consensus, as well as an updated international guideline on the approach of these patients is required for the improvement of physician care.

Adina Roman $^{1,2}$, Anca-Meda Georgescu ${ }^{2,3}$, Septimiu Moldovan ${ }^{4}$, Mircea Stoian $^{2,4}$, Daniela Dobru ${ }^{1,2}$

1) Gastroenterology Department, Mureș County Clinical Hospital, Târgu Mureș; 2) George Emil Palade University of Medicine, Pharmacy, Sciences and Technology, Târgu Mureș; 3) Infectious Diseases Department, Mureș County Clinical Hospital, Târgu Mureș; 4) Intensive Care Unit Department, Mureș County Clinical Hospital, Târgu Mureș, Romania

Correspondence: Adina Roman, adina.roman91@gmail.com

Conflicts of interest: None.

Acknowledgments: We would like to thank the entire personnel involved in the care of patients suffering from COVID-19 at Mureș County Clinical Hospital and for their dedication.

DOI: $10.15403 /$ jgld-3119

\section{REFERENCES}

1. Xin $\mathrm{S}, \mathrm{Xu}$ J, Yu Y. Abnormal Liver Function Tests of Patients with Coronavirus Disease 2019 in Mainland China: A Systematic Review and Meta-Analysis. J Gastrointestin Liver Dis. 2020;29:219-226. doi:10.15403/jgld-2513

2. Worldometer. Covid-19 coronavirus pandemic. November 2020 Available at: https://www.worldometers.info/coronavirus/\#countries

3. Qi X, Liu C, Jiang Z, et al. Multicenter analysis of clinical characteristics and outcomes in patients with COVID-19 who develop liver injury. J Hepatol 2020;73:455-458. doi:10.1016/j.jhep.2020.04.010

4. Cai Q, Huang D, Yu H, et al. COVID-19: abnormal liver function tests. J Hepatol 2020;73:566-574. doi:10.1016/j.jhep.2020.04.006
5. Boettler T, Newsome PN, Mondelli MU, et al. Care of patients with liver disease during the COVID-19 pandemic: EASL-ESCMID position paper. JHEP Rep 2020;2:100113. doi:10.1016/j.jhepr.2020.100113

6. Ye Z, Song B. COVID-19 related liver injury: call for international consensus. Clin Gastroenterol Hepatol 2020;18:2848-2851. doi:10.1016/j.cgh.2020.05.013

\section{Occurrence and recurrence of hepatocellular carcinoma in patients with HCV genotype $1 \mathrm{~b}$ related cirrhosis treated with Ledipasvir + Sofosbuvir \pm Ribavirin}

\section{To the Editor,}

Although direct antiviral agents (DAAs) therapy for hepatitis $\mathrm{C}$ virus (HCV) has been revolutionary, with a sustained virologic response (SVR) rate of more than $90 \%$, with favorable effects on improving liver function and reducing cirrhosis complications, the impact of treatment on de novo or recurrent hepatocellular carcinoma (HCC) development is still debated $[1,2]$.

The aim of this study was to evaluate the prevalence and risk factors for occurrence and recurrence of HCC in patients with $\mathrm{HCV}$ genotype $1 \mathrm{~b}$ related cirrhosis treated with Ledipasvir + Sofosbuvir \pm Ribavirin (LDV/SOF \pm RBV). We conducted a multicentric retrospective cohort study on 349 patients with decompensated liver cirrhosis due to $\mathrm{HCV}$ infection genotype $1 \mathrm{~b}$ treated with $\mathrm{LDV} / \mathrm{SOF} \pm \mathrm{RBV}$ for 12-24 weeks from September 2018 to September 2019. The median follow-up period was 20 months (5-24).

In this cohort, 18 subjects were previously treated for HCC (transarterial chemoembolization in 10 cases, surgery in 4 and radiofrequency ablation in 4). None of these patients was coinfected with hepatitis B virus (HBV). Patients were eligible for DAAs therapy if they had no imaging sign of HCC recurrence 3 months after the HCC treatment. At the beginning of DAAs treatment, the median viremic load was 196,000 IU/ $\mathrm{mL}(1,210 \div 6,590,000 \mathrm{IU} / \mathrm{mL})$ and SVR12 was obtained in 12 (66.7\%). The HCC recurrence appeared in 8 subjects (44.4\%), 6 of them (75\%) with HCC BCLC stage A and 2 (25\%) with HCC BCLC stage B. The median time elapsed between the last HCC procedure and the start of DAAs was 20 months (3-60), between last procedure for HCC and recurrence 25 months (13-39) and between start of DAAs therapy and HCC recurrence 12 months (6-12). The proportion of patients with HCC recurrence since the start of DAAs therapy is represented in Supplementary file. Four patients (22.2\%) died during the follow up period.

The SVR rate was significantly lower in the subgroup of patients with recurrence compared with those without recurrence ( $50 \%$ vs. $80 \%, p=0.034)$. No statistically significant differences were obtained for gender, age, interval between HCC diagnosis and initiation of DAAs therapy, alphafetoprotein value and MELD score (Supplementary file).

The second analysis focused on HCC occurrence was conducted on 321 subjects (12 cases stopped the therapy to severe adverse reactions), divided in two subgroups: without HCC diagnosis during follow-up (306 patients) and with HCC occurrence (15 individuals). Surveillance for HCC was made 
according to guidelines: liver ultrasound with alpha fetoprotein every 6 months [3].

After DAAs treatment, patients significantly improved their liver function. (Supplementary file). HCC occurred de novo in 15 out of 321 (4.7\%) subjects. In the subgroup of HCC occurrence compared with the subgroup without HCC, a significantly lower value of platelet count $\left(56 \times 10^{9} / \mathrm{L}\right.$ vs $\left.95 \times 10^{9} / \mathrm{L}, \mathrm{p}=0.001\right)$, as well as albumin levels $(2.8 \mathrm{~g} / \mathrm{dL}$ vs $3.4 \mathrm{~g} / \mathrm{dL}, \mathrm{p}=0.001$ ), a significantly higher level of bilirubin ( $2.64 \mathrm{mg} / \mathrm{dL}$ vs. $1.6 \mathrm{mg} / \mathrm{dL}, \mathrm{p}=0.014)$ were noted. There were no significant differences regarding the baseline clinical and biological parameters, except the Child-Pugh score (significantly higher in the subgroup of patients with de novo HCC). There were no differences in the response to DAAs, with a similar SVR12 rate between the two groups (76.9\% vs $89.2 \%, \mathrm{p}=0.171$ ) (Supplementary file).

Our results regarding the HCC recurrence rate was higher than expected, $44.4 \%$ (30.6/100 patient-year) and the SVR rate was only $66.7 \%$, as we included only patients with decompensated cirrhosis. The HCC-DAAs interval was between 3 to 60 months. Most of the authors recommend a delay of at least 6 months of DAAs therapy after HCC complete response [4-6]. Reig et al. [7] found $27.6 \%$ recurrence rate of for a median follow-up of 5.7 months, higher than expected, with a median period of 3.5 months between treatment and HCC recurrence. Cardoso et al. [8] found a significantly lower rate of HCC recurrence (7.4\%), but still higher than expected, with a median follow-up of 12 months. All the studies mentioned above had a SVR rate higher than 90\%. Idilman et al. [9] obtained similar results: $48.5 \%$ recurrence rate of $\mathrm{HCC}$ with a median follow-up of 6 months after treatment and a statistically significant period of time between HCC diagnosis and DAAs treatment (24 months without recurrence vs. 9 months with recurrence), that may explain the higher recurrence rate. Our HCCDAAs interval was 20 months in the patients with no HCC recurrence versus 13.5 in those recorded with HCC recurrence $(\mathrm{p}=\mathrm{NS})$.

In our analysis, the lack of SVR did not predict the occurrence of a HCC; instead, we found similar predictive factors with the data from the literature [10]: poor liver function [high Child score ( $\mathrm{p}=0.007)$, low albumin $(\mathrm{p}=0.001)$ and high bilirubin levels $(\mathrm{p}=0.01)$. The DAAs - HCC interval was 3-18 months, with a median length of 6 months.

In conclusion, the recurrence rate of HCC in cirrhotic patients treated with $\mathrm{LDV} / \mathrm{SOF} \pm \mathrm{RBV}$ was $44.4 \%(30.6 / 100$ patient-year). The occurrence rate of HCC was $4.7 \%$ in a median follow-up period of 20 months. The predictive factors for the HCC occurrence were a lower platelet number and an impaired liver function.

Corina Silvia Pop ${ }^{1}$, Carmen Monica Preda ${ }^{2}$, Mircea Manuc ${ }^{2}$, Liliana Simona Gheorghe ${ }^{2}$, Doina Istratescu ${ }^{3}$, Andreea Elena Chifulescu ${ }^{3}$, Theodor Voiosu ${ }^{4}$, Mircea Diculescu ${ }^{2}$, Cristian Tieranu ${ }^{5}$, Laura Iliescu $^{6}$

1) Carol Davila University of Medicine and Pharmacy, Internal Medicine Department, Emergency University Hospital, Bucharest; 2) Carol Davila University of Medicine and Pharmacy, Gastroenterology\&Hepatology Department, Clinic Fundeni Institute,
Bucharest; 3) Gastroenterology\&Hepatology Department, Clinic Fundeni Institute, Bucharest; 4) Carol Davila University of Medicine and Pharmacy, Internal Medicine Department, Colentina Hospital, Bucharest; 5) Carol Davila University of Medicine and Pharmacy, Gastroenterology\&Hepatology Department, Elias Emergency Hospital, Bucharest; 6) Carol Davila University of Medicine and Pharmacy, Internal Medicine Department, Clinic Fundeni Institute, Bucharest, Romania

Correspondence: Carmen Monica Preda, carmenmonica.preda@gmail.com

Conflicts of interest: L.S.G., L.I., M.D. received honoraria and educational grants from Gilead. The other authors declare no conflicts of interest.

DOI: $10.15403 /$ jgld-3148

Supplementary material: To access the supplementary material visit the online version of the J Gastrointestin Liver Dis at http://dx.doi. org $/ 10.15403 /$ jgld-2878

\section{REFERENCES}

1. Gheorghe LS, Preda C, Iliescu L, et al. Efficacy and Safety of Ledispavir/ Sofosbuvir with or without Ribavirin in patients with Decompensated Liver Cirrhosis and Hepatitis C Infection: a Cohort Study. J Gastrointestin Liver Dis 2020;29:385-390. doi:10.15403/jgld-2448

2. Preda CM, Popescu CP, Baicus C, et al. Real-world efficacy and safety of ombitasvir, paritaprevir/r+dasabuvir+ribavirin in genotype $1 \mathrm{~b}$ patients with hepatitis C virus cirrhosis. Liver Int 2018;38:602-610. doi:10.1111/ liv. 13550

3. Marrero JA, Kulik LM, Sirlin CB, et al. Diagnosis, staging and management of hepatocellular carcinoma: 2018 practice guidance by the American Association for the study of Liver Diseases. Hepatology 2018;68:723-750. doi:10.1002/hep.29913

4. Piñero F, Mendizabal M, Ridruejo E, et al. Treatment with directacting antivirals for $\mathrm{HCV}$ decreases but does not eliminate the risk of hepatocellular carcinoma. Liver Int 2019;39:1033-1043. doi:10.1111/ liv. 14041

5. Preda CM, Baicus C, Sandra I, et al. Recurrence rate of hepatocellular carcinoma in patients with treated hepatocellular carcinoma and hepatitis $\mathrm{C}$ virus-associated cirrhosis after ombitasvir/paritaprevir/ ritonavir+dasabuvir+ribavirin therapy. United European Gastroenterol J 2019;76:699-708. doi:10.1177/2050640619841254

6. Ji F, Yeo YH, Wei MT, et al. Sustained virologic response to directacting antiviral therapy in patients with chronic hepatitis $\mathrm{C}$ and hepatocellular carcinoma: A systematic review and meta-analysis. J Hepatol 2019;71:473-485. doi:10.1016/j.jhep.2019.04.017

7. Reig M, Mariño Z, Perelló C, et al. Unexpected high rate of early tumor recurrence in patients with HCV-related HCC undergoing interferonfree therapy. J Hepatol 2016;65:719-726. doi:10.1016/j.jhep.2016.04.008

8. Cardoso H, Vale AM, Rodrigues S, et al. High incidence of hepatocellular carcinoma following successful interferon-free antiviral therapy for hepatitis C associated cirrhosis. J Hepatol 2016;65:1070-1071. doi:10.1016/j.jhep.2016.07.027

9. Idilman R, Demir M, Aladag M, et al. Low recurrence rate of hepatocellular carcinoma following ledipasvir and sofosbuvir treatment in a real-world chronic hepatitis C patients cohort. J Viral Hepat 2019;26:666-674. doi:10.1111/jvh.13075 
10. Yoshimasu Y, Furuichi Y, Kasai Y, et al. Predictive factors for hepatocellular carcinoma occurrence or recurrence after direct-acting antiviral agents in patients with chronic hepatitis C. J Gastrointestin Liver Dis 2019;28:63-71. doi:10.15403/jgld.2014.1121.281.hpc

\section{Fighting Hepatitis C in a COVID-19 ecosystem. Public health policies are needed more than ever}

\section{To the Editor,}

One of the most important lessons that National Health Systems have learned with the coronavirus disease 2019 (COVID-19) pandemic was that more than clinical measures, diseases that depend on human behavior are primarily fought with targeted Public Health Policies (PHP) [1]. All the countries that deviated from this approach have registered the worst results [2]. In the opposite direction, those that most vigorously resorted to structured forms and with an effective transfer of concepts to the field were the ones that have been obtaining better results.

Hepatitis $C$ is also one of the diseases in which its transmission depends the most on adopted behaviors of oneself and among communities [e.g., persons who inject drugs (PWID), prisoners, etc.].

The LEHC Project approach is to focus on PHP towards achieving the hepatitis $C$ elimination by 2030 , as proposed by the Sustainable Development Goals [3]. This project encompasses an epidemiological model based on five populations (PWID, prisoners, vertical transmission, blood products, remaining population) [4]. Each population group has specific dynamics and epidemiological history, with specific reactions to each of the twenty-four PHP focused on hepatitis $\mathrm{C}$ that were considered in the LEHC project. Modelling results were obtained by considering different implementation levels of each PHP, integrating the classic Markov Chain epidemiological mathematical along with a statistical one based on Adaptive Conjoint Analysis (ACA) that results from the National Experts reading about the impact of each PHP in a national population.

The LEHC project allows to simulate different PHP implementation levels, for each year until 2030, forecasting results in each of the five populations and the country's total population. This model has been progressively implemented in a group of ten European countries, chosen for the diverse characteristics among them, with five being already completed: Bulgaria, Romania, Portugal, Spain and Austria.

For the total population in Bulgaria, it is estimated that in 2019 there were close to 67,800 hepatitis $C$ cases (distributed by: PWID=42,200; prisoners 6,200; blood products 1,600 ; remaining population 17,800$)$.

According to the LEHC model, Bulgaria can further intensify eleven PHP measures. Focusing on PHP related to Awareness and Prevention, as well as Testing and Diagnosis, it would be possible to have the most impact in reducing HCV infection cases in Bulgaria. Despite Bulgaria's broad resource allocation and current PHP directed to eliminate hepatitis $\mathrm{C}$ by 2030 , results from the LEHC model suggest that the country will not achieve the goal established by WHO in that year. However, by testing the implementation of all the PHP considered in the LEHC project, forecasts suggest that the elimination goal can be attained as soon as 2029. Further implementing PHP to shorten the necessary time to achieve the HCV elimination goal has also been verified in a recent published article about the LEHC model application in Romania [5]. This reading is also aligned with the obtained results for the remaining countries with complete LEHC modelling, with corresponding articles being also under submission.

Modelled data were produced previously to the COVID-19 pandemic. We see with enormous concern that hepatitis $\mathrm{C}$ may be one of the most punished diseases by COVID-19: the drastic reduction on the number of aerosol generating procedures across Europe; human, monetary and technical resources are diverted to assist the COVID-19 emergency, being known that hepatitis $\mathrm{C}$ is formed mainly by stigmatized populations, hence where decision-making powers are more prone to decrease investment without great public impact. Therefore, we consider that COVID-19 is a huge threat to the fulfillment of the WHO elimination goal for hepatitis $\mathrm{C}$, which may only be countered by a strong defense of what was already positively worked.

Henrique Lopes ${ }^{1}$, Ricardo Baptista-Leite ${ }^{2}$, Diogo Franco ${ }^{1}$, Todor Kundurzhiev ${ }^{3}$, Lyudmila Mateva ${ }^{4}$, Dejan Jelev ${ }^{4}$, Marieta Simonova ${ }^{5}$

1) Public Health Unit, Institute of Health Sciences, Catholic University of Portugal, Lisbon, Portugal; 2) Faculty of Health, Medicine and Life Sciences, Maastricht University, Institute of Health Sciences, Catholic University of Portugal, Lisbon, Portugal; 3) Biostatistics and Medical Informatics, Department of Occupational Medicine. Faculty of Public Health, Medical University, Sofia. Bulgaria; 4) Clinic of Gastroenterology, St. Ivan Rilski University Hospital, Chair of Internal Medicine, Faculty of Medicine, Medical University, Sofia. Bulgaria; 5)Clinic of Gastroenterology, Department of Gastroenterology, HPB Surgery and Transplantology, Military Medical Academy, Sofia, Bulgaria.

Correspondence: Prof. Henrique Lopes, henrique.lopes@ucp.pt

Conflict of interest: None.

Acknowledgements: Gilead Sciences Europe Ltd provided financial support in 2017 for national data collection part of the modeling, web-design and IT of the LEHC project.

DOI: 10.15403 /jgld-2794

\section{REFERENCES}

1. Davies NG, Kucharski AJ, Eggo RM, et al. Effects of non-pharmaceutical interventions on COVID-19 cases, deaths, and demand for hospital services in the UK: a modelling study. Lancet Public Health 2020;5:e375-e385. doi:10.1016/S2468-2667(20)30133-X

2. Rawaf S, Yamamoto HQ, Rawaf D. Unlocking towns and cities: COVID-19 exit strategy. East Mediterr Health J 2020;26:499-502. doi:10.26719/emhj.20.028

3. Waheed Y, Siddiq M, Jamil Z, Najmi MH. Hepatitis elimination by 2030: Progress and challenges. World J Gastroenterol. 2018;24:4959-4961. doi:10.3748/wjg.v24.i44.4959 
4. Baptista-Leite R, Lopes H, Wandewalle B, Félix J, Franco D. Preprint USP-ICS 2019 /7 - Project LEHC - Public Health Policies impact in HCV epidemiological modeling: a tool towards a micro-elimination path. 2019. doi:10.13140/RG.2.2.32204.77443/1
5. Lopes H, Baptista-Leite R, Franco D, et al. Modeling the Puzzle of Hepatitis C Epidemiology in Romania: A Pathway to Control. J Gastrointestin Liver Dis 2020;29:377-384. doi: 10.15403/jgld-643 